\title{
Editorial
}

\section{Possible roles of endogenous plant viral sequences and transgenes containing viral sequences in both virus resistance and virus emergence}

\author{
Pierre-Yves TEYCHENEY ${ }^{1}$ and Mark TEPFER ${ }^{2}$ \\ 1 CIRAD-UPR75 - Station de Neufchâteau, Sainte-Marie, 97130 Capesterre Belle-Eau, Guadeloupe, France \\ E-mail: teycheney@cirad.fr \\ 2 Plant Virology Group, ICGEB Biosafety Outstation, Via Piovega, 23, 31056 Ca’ Tron di Roncade, Italy
}

One of the communication tools of the EC-funded Biosafenet project is a series of six Biosafenet Seminars that will be held 2007-2009. Their purpose is to convene small groups of scientists to consider emerging issues related to the potential impact of genetically modified organisms (GMOs). The first Seminar was organized by the ICGEB at the MasterCampus of the Fondazione Cassamarca in Ca' Tron di Roncade (Italy) on 6th-8th June 2007, and focused on the potential impact of both naturally occurring and transgenic viral sequences in plant genomes.

Viral sequences have been introduced into plant genomes to create virus resistant transgenic plants (VRTPs) since the mid-1980s. More recently, it has been shown that fragments of certain viral genomes have been naturally inserted into plant genomes and maintained through evolution (Harper et al., 2002; Hull et al., 2000). The current research on what can be considered to be natural analogs of viral transgenes is focused primarily on sequences derived from dsDNA viruses, the pararetroviruses of the family Caulimoviridae. The endogenous pararetroviral sequences (EPRVs) that have been described so far are often highly degenerate, but in some cases can be activated to produce infectious virus, and thus disease (Staginnus and Richert-Pöggeler, 2006). For ssDNA viruses, represented by the family of Geminiviridae, only fragments of homologous sequences have been reported in the genome of Nicotiana spp (Murad et al., 2004). The expression and regulation of VRTPs and EPRVs involve similar transcriptional and post-transcriptional pathways (see Fig. 1). Moreover, both VRTPs and EPRVs have the potential to contribute to the emergence of new virus populations. Therefore, questions about both types of inserted viral sequences arise: do both confer resistance by similar mechanisms in certain cases? Could both types of inserted sequences contribute to the emergence of new viral genomes?

In order to help answer these questions, the first Biosafenet Seminar focused on the convergence between what are to date two distinct areas of research: (1) EPRVs, for which it is now established that they can lead to virus emergence (Staginnus and Richert-Pöggeler, 2006), while the evidence concerning their role in virus resistance is less evident (Mette et al., 2002), and (2) VRTPs expressing viral sequences, for which there is a wealth of information on resistance mechanisms, and little information regarding their potential role in virus emergence (Prins et al., in press). The workshop brought together 27 researchers from 11 countries, most of whom work on EPRVs or VRTPs, with a few scientists working in related research areas, such as virus evolution and endogenous viral sequences of mammals and insects.

Several talks provided participants with an overview of the organization and expression of viral sequences integrated in the genomes of plants, insects and mammals. A striking feature of plant EPRVs, whether infectious or not, is that they are present mostly in heterochromatin, and that their integration sites are often flanked with transposable elements in the family Metaviridae. Another feature shared by the non-infectious EPRVs characterized so far is that they are transcribed at low levels into RNA molecules that often trigger resistance based on transcriptional or post-transcriptional gene silencing mechanisms (Hohn et al., 2007). Strikingly, similar mechanisms also play a central role in the virus resistance conferred by the expression of viral transgenes in many - but not all VRTPs. 


\section{Resistance and viral emergence from a viral transgene}

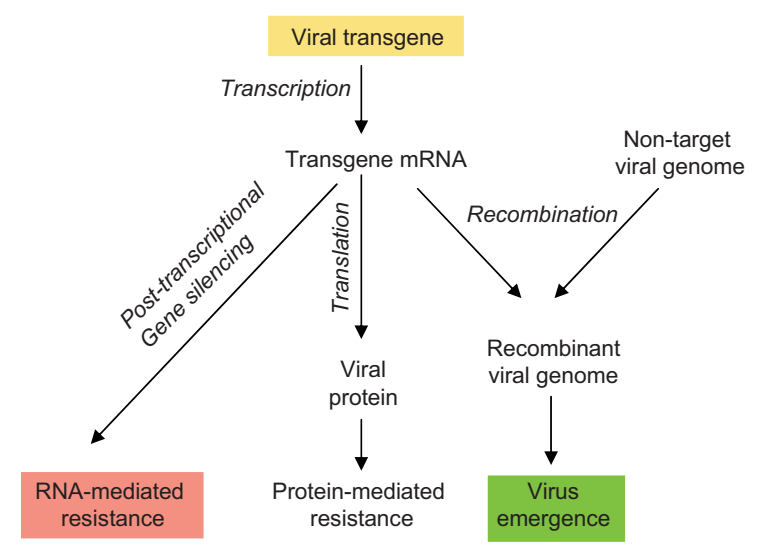

\section{Resistance and viral emergence from an EPRV}

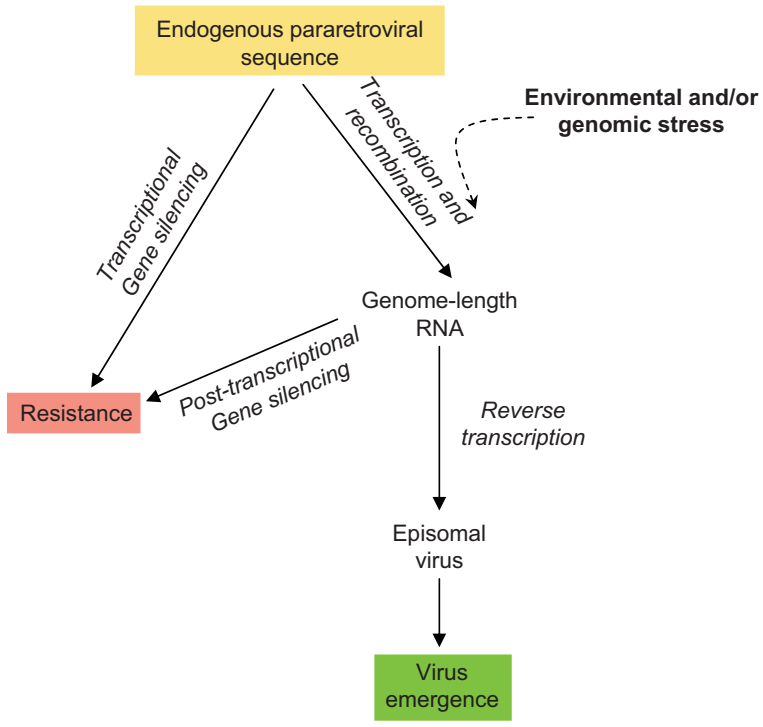

Figure 1. Pathways that could potentially lead to virus resistance and virus emergence from viral transgenes and endogenous pararetroviral sequences. In both cases, the starting point is a viral sequence inserted in the plant genome (yellow boxes), and it has been shown that gene silencing induced by the integrated sequence can lead to virus resistance (red boxes). Certain EPRVs can lead to emergence of infectious virus (green boxes) through steps involving release from gene silencing, recombination, and transcription. In contrast, in VRTPs, although recombination between transgene mRNAs and viral RNAs has been well described, there is no evidence that this leads to emergence of novel recombinant viruses.

An important point that was reinforced at this meeting is that although VRTPs and EPRVs may confer virus resistance via shared common mechanisms, they differ substantially in the mechanisms by which they could be involved in the emergence of new viruses. It has been shown that the RNA transcribed from viral transgenes can recombine with the RNA of an infecting viral genome. However, there is no evidence that this leads to recombinant viruses other than those occurring in non-transgenic plants infected simultaneously with two viruses, which is a common phenomenon. New results presented in $\mathrm{Ca}$ ' Tron showed that under controlled experimental conditions, equivalent populations of recombinant viruses occurred in transgenic and non-transgenic plants (Turturo et al., in press). In contrast, the emergence of viral genomes from EPRVs has been shown to result from stress-induced activation of infectious EPRVs, not from recombination events involving EPRVs and infecting viruses (Richert-Pöggeler et al., 2003). Recent work shows that epigenetic changes in plant chromatin could promote heritable adaptive changes, particularly in response to environment changes. This provides exciting clues about the expression of infectious EPRVs from highly rearranged integrated copies that would require multiple recombination events for being expressed. In this regard, the expression of infectious EPRVs re- lates to that of animal endogenous retroviruses such as HERV-K, for which it has been shown that a putative ancestral progenitor led to the production of functional and infectious viral particles that amplify via an extracellular pathway involving re-infection of neighboring cells.

Recombination events between endogenous animal viral sequences and incoming viral genomes are not documented, although in silico analyses of nucleotide sequences show that such endogenous viral sequences are widespread in insect genomes. Recent work shows that insect and viral genomes exchange genetic material, and that antiviral mechanisms might result from endogenous viral sequences, therefore protecting insect hosts against infections by cognate viruses. In certain other cases, insect endogenous viral sequences are also beneficial to their hosts, since they encode functions that are necessary to the colonization of hosts by insect larvae. Whether the presence of endogenous viral sequences in eukaryotic genomes influences the evolution of virus populations other than through virus resistance mechanisms remains unknown.

At the end of this Biosafenet Seminar, a much clearer picture of the similarities and differences between factors regulating the behavior of VRTPs and EPRVs had emerged. In a final discussion of topics that merit particular efforts in the near future, there was a clear consensus 
that a key question - and one that concerns both types of inserted viral sequences - is the role of biotic, abiotic and genomic stresses on the behavior of the inserted viral sequences. There are well known effects of biotic and abiotic stress on post-transcriptional gene silencing, which could have effects on either virus resistance in VRTPs and/or on EPRV emergence. It is also quite clear that genomic stresses have even more far-reaching and less well understood effects leading to changes - sometimes heritable - in both transcriptional and post-transcriptional gene silencing. In the broader context of genome/environment interactions, research in this area will certainly cast light on the timely issue of the impact of stress-related effects of ongoing climate changes on genomes' plasticity and expression, and how these could impact both VRTPs and EPRVs. European scientists have been consistently on the cutting edge of this research area, which the participants felt is exceptionally attractive for further and enhanced support at the international level.

\section{REFERENCES}

Harper G, Hull R, Lockhart B, Olszewski N (2002) Viral sequences integrated into plant genomes. Annu. Rev. Phytopathol. 40: 119-136

Hohn T, Richert-Pöggeler K, Staginnus C, Harper G, Schwartzacher T, Teo C-H, Teycheney P-Y, IskraCaruana ML, Hull R (2007) Evolution of integrated plant viruses. In Roossinck M, ed, Plant virus evolution, Springer (in press)

Hull R, Harper G, Lockhart B (2000) Viral sequences integrated into plant genomes. Trends Plant Sci. 5: 362-365

Mette MF, Kanno T, Aufsatz W, Jakowitsch J, van der Winden J, Matzke MA, Matzke AJ (2002) Endogenous viral sequences and their potential contribution to heritable virus resistance in plants. EMBO J. 21: 461-469

Murad L, Bielawski JP, Matyasek R, Kovarik A, Nichols RA, Leitch AR, Lichtenstein CP (2004) The origin and evolution of geminivirus-related DNA sequences in Nicotiana. Heredity 92: 352-358

Prins M, Laimler M, Noris E, Schubert J, Wassenegger M, Tepfer M (in press) Strategies for antiviral resistance in transgenic plants. Molec. Plant Pathol.

Richert-Pöggeler KR, Noreen F, Schwarzacher T, Harper G, Hohn T (2003) Induction of infectious petunia vein clearing (pararetro) virus from endogenous provirus in petunia. EMBO J. 22: 4836-4845

Staginnus C, Richert-Pöggeler KR (2006) Endogenous pararetroviruses: two-faced travelers in the plant genome. Trends Plant Sci. 11: 485-491

Turturo C, Friscina A, Gaubert S, Jacquemond M, Thompson JR, Tepfer M (in press) Evaluation of the potential risks associated with recombination in transgenic plants expressing viral sequences. J. Gen. Virol. 